\title{
Novel mutations in the human elastin gene $(E L N)$ causing isolated supravalvular aortic stenosis
}

\author{
SEONMIN PARK ${ }^{1}$, EUL-JU SEO ${ }^{1}$, HAN-WOOK YOO $^{1}$ and YOUNGHO KIM ${ }^{2}$ \\ ${ }^{1}$ Genome Research Center for Birth Defects and Genetic Disorders, University of Ulsan College of Medicine, \\ Asan Medical Center, 388-1, Pungnap-2 Dong, Songpa-Gu, Seoul 138-736; ${ }^{2}$ Department of Biochemistry, \\ Wonkwang University, School of Medicine, 344-2, Sinyong-Dong, Iksan, Jeollabuk-Do 570-749, Korea
}

Received January 9, 2006; Accepted April 5, 2006

\begin{abstract}
Supravalvular aortic stenosis (SVAS), an inherited vascular disease, is caused by mutations in the elastin gene $(E L N)$. Our aim was to identify novel mutations of $E L N$ and to determine the expression of $E L N$ in patients with SVAS. For screening mutations in $E L N$, we performed PCR-directed sequence analysis with genomic DNA isolated from SVAS patients and control subjects. Expression of ELN at the mRNA and protein levels were assessed by real-time PCR and Western blot analyses, respectively, using primary skin fibroblast cultures established from SVAS patients and control subjects. We identified two novel mutations of ELN, G297_A308del and Q700X, in two unrelated Korean patients with isolated SVAS. G297_A308del occurred de novo while Q700X was derived maternally. In the patient with G297_A308, elastin expression was not significantly altered at the mRNA level, but was reduced to $\sim 50 \%$ of the normal control at the protein level. The elastin expression levels in the patient with Q700X were reduced to $<50 \%$ of the normal controls at both the mRNA and protein levels. Our findings confirm that functional haploinsufficiency of elastin is responsible for the pathogenesis associated with isolated SVAS across different ethnic backgrounds.
\end{abstract}

\section{Introduction}

Supravalvular aortic stenosis (SVAS; OMIM 185500) is an inherited vascular disease characterized by localized or diffuse

Correspondence to: Dr Youngho Kim, Department of Biochemistry, Wonkwang University, School of Medicine, 344-2, Sinyong-Dong, Iksan, Jeollabuk-Do 570-749, Korea

E-mail: youngkim@wonkwang.ac.kr

Dr Han-Wook Yoo, Genome Research Center for Birth Defects and Genetic Disorders, University of Ulsan College of Medicine, Asan Medical Center, 388-1, Pungnap-2 Dong, Songpa-Gu, Seoul 138-736, Korea

E-mail: hwyoo@amc.seoul.kr

Key words: congenital defects, elastin, extracellular matrix, supravalvular aortic stenosis narrowing of the ascending aorta primarily caused by thickening of the aorta media (1). SVAS occurs as an isolated vascular disease or as part of Williams-Beuren syndrome (WBS; OMIM 1945050), a developmental disorder associated with cardiovascular, neurobehavioral, facial, and metabolic abnormalities (2). WBS is caused by a $1.5-2 \mathrm{Mb}$ deletion at chromosome $7 \mathrm{q} 11.2$ that involves the elastin gene $(E L N)$ and at least 15 other genes $(3,4)$, whereas SVAS is caused by mutations in ELN (5-9). So far, 43 mutations in ELN have been identified in patients with isolated SVAS, including substitution, splicing, regulatory, deletion, insertion and rearrangement mutations (The Human Gene Mutation Database, www.hgmd.org).

Elastin is the major extracellular matrix protein deposited by vascular smooth muscle cells in the arterial media, contributing up to $\sim 50 \%$ of the vessel's dry weight $(10,11)$. Elastin is secreted as tropoelastin, a soluble monomer of elastin, and then, after a series of post-translational modifications, is crosslinked by lysyl oxidase into insoluble elastin polymers. In the arteries, the elastin polymers form elastic lamella around the arterial lumen, providing resilience against the hemodynamic stresses of cardiac systole and diastole $(10,11)$.

Herein, we report novel mutations of ELN, G297_A308del and Q700X, from two unrelated Korean patients with isolated SVAS. Using real-time PCR and Western blot analyses, we have demonstrated that both mutations result in significantly reduced expression of $E L N$ at the protein level, confirming that functional haploinsufficiency of elastin is responsible for the pathogenesis of isolated SVAS across different ethnic backgrounds.

\section{Materials and methods}

Patients. Diagnosis of patients with SVAS and unaffected mutation carriers was based on physical and echocardiographic examinations. Using fluorescent in situ hybridization (FISH) analysis with a WBS critical region probe (Vysis), all SVAS patients were also diagnosed at a molecular level. All individuals included in this study, both affected and unaffected, are of Korean origin.

Mutation analysis. After informed consent, blood samples were obtained from patients and control subjects. Genomic DNA from two families with SVAS and 100 healthy control subjects 
were extracted from whole blood using a Puregene DNA isolation kit according to the manufacturer's protocol (Gentra Systems). A total of 34 exons and 68 intron-exon boundaries in $E L N$ were PCR-amplified with primers specific to the flanking regions of each exon and then sequenced with the same primers using a cycle sequencing ready reaction kit (Applied Biosystems). The sequences of primers are available upon request. Scanning and analysis of the sequences were performed using an ABI 3100 genetic analyzer (Applied Biosystems). The nucleotide numbering used in this study reflects the coding region of ELN as previously described (8).

Verification of the G297_A308del mutation. Exon 17 of ELN was amplified from the genomic DNA of 100 healthy control subjects with primers specific to the flanking sequences of exon 17. The sequences of forward and reverse primers were 5'-CTGGCGTGCCTGGGGCAATTCCTG-3' and 5'-TTG CGGCTAGGGTCTCCGAGGTC-3', respectively. The PCR amplifications were done using a cycle sequencing ready reaction kit (Applied Biosystems) according to the manufacturer's protocol. The sizes of PCR amplicons were determined by $7.5 \%$ PAGE.

Real-time PCR analysis. Primary skin fibroblast cultures were established from patients with SVAS and control subjects as previously described (12). Total RNA was isolated from cultured skin fibroblasts using an RNAzolB solution (Bioprobe). The cDNA was synthesized with $1 \mu \mathrm{g}$ of total RNA using a SuperScript first strand synthesis kit (Invitrogen) according to the manufacturer's protocol. Commercially available $E L N$ and $\beta$-actin probes (TaqMan ${ }^{\circledR}$ gene expression assays; Applied Biosystems) were used for real-time PCR analysis. Amplifications were carried out in triplicate using a TaqMan Universal PCR Master mix (Applied Biosystems) according to the manufacturer's suggestions. Data analysis was performed using ABI PRISM 7000 software, version 1.1 (Applied Biosystems).

Western blot analysis. The cultured skin fibroblasts were harvested at confluence and lyzed in a buffer containing $1 \%$ Triton X-100, $0.35 \mathrm{M} \mathrm{NaCl}, 10 \%$ glycerol, $1 \mathrm{mM}$ EDTA, $1 \mathrm{mM}$ PMSF and $50 \mathrm{mM}$ Tris- $\mathrm{HCl}, \mathrm{pH}$ 8.0. The protein concentrations of the skin fibroblast lysates were determined using a BCA protein assay kit (Pierce). Total protein $(\sim 70 \mu \mathrm{g})$ was used for Western blot analysis with a rabbit anti-elastin antibody (Calbiochem).

\section{Results}

Identification of novel mutations of ELN in patients with isolated SVAS. Using PCR-directed sequence analysis of all coding sequences of $E L N$, we identified an in-frame deletion mutation G297_A308del and a nonsense mutation Q700X in two unrelated patients with isolated SVAS. Using FISH analysis with a probe specific to the WBS critical region at chromosome 7q11.2, we confirmed that neither ELN allele was deleted in any of the SVAS patients tested.

The G297_A308del mutation was detected in a one-yearold boy who presented with isolated SVAS, but did not show any abnormalities in facial features or intelligence. Neither of
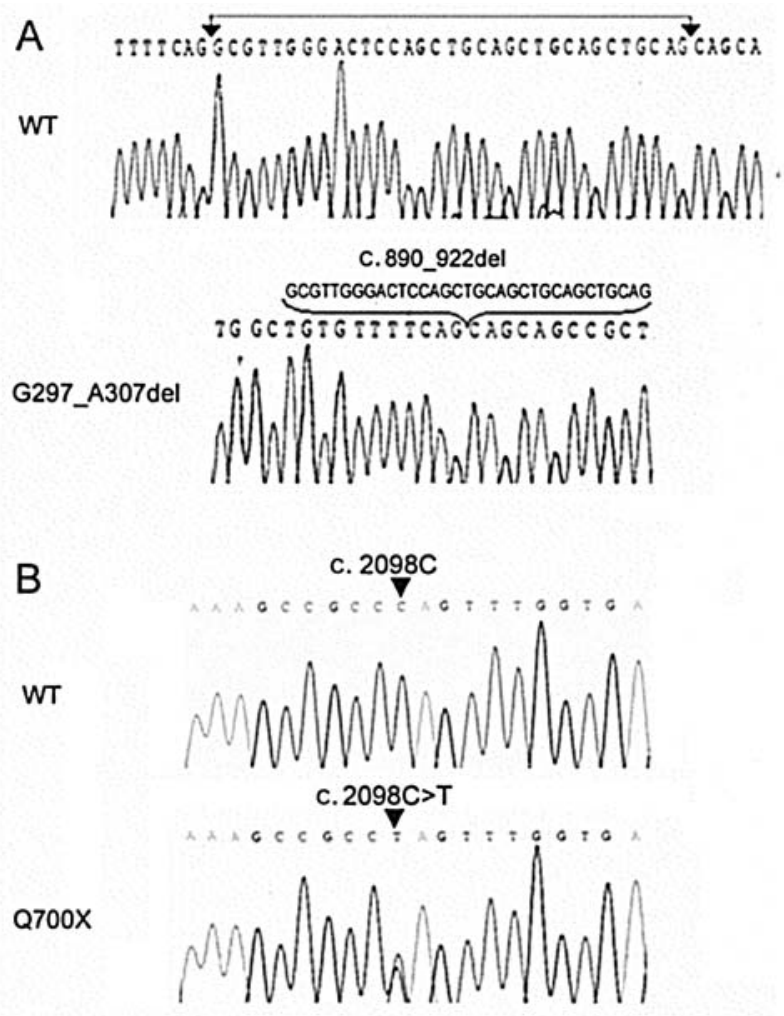

Figure 1. Sequence analysis of the wild-type (WT) and mutant alleles. (A) The G297_A308del mutation. The deleted sequences in the mutant allele are indicated by an arrowed line in the WT allele. (B) The Q700X mutation. The mutated sequence is indicated by an arrowhead.

his parents carried the G297_A308del mutation, indicating that this mutation occurred de novo. The G297_A308del mutation was caused by a 33-bp deletion at nucleotide positions 890-922 in exon 17 (Fig. 1A), resulting in a deletion of 11 amino acids at codons 297-307.

Another one-year old boy who suffered from SVAS and peripheral pulmonary stenosis (PPS) carried a nonsense mutation Q700X caused by a nucleotide substitution 2098C $>$ T in exon 29 (Fig. 1B). In addition to SVAS and PPS, this patient also showed right thumb polydactyly and a narrow palpebral fissure. The patient's mother carried the same mutation, but was asymptomatic for SVAS, indicating low phenotypic penetrance of the mutation in this family.

Verification of the G297_A308del mutation. To verify that G297_A308del was a mutation, we genotyped 200 alleles of healthy control subjects. In PCR analysis of exon 17 of ELN, the patient with G297_A308del showed a biallelic pattern with a 203-bp fragment and a 236-bp fragment, whereas healthy controls presented only a 236-bp fragment (Fig. 2), confirming that G297_A308del is not a polymorphism. The patients' parents showed a monoallelic pattern with a 236-bp fragment (Fig. 2), indicating that G297_A308del was a de novo mutation.

Expression analysis of elastin in the SVAS patients. To study the expression of elastin in SVAS patients, we generated primary skin fibroblast cultures from a healthy control subject and from patients carrying the G297_A308del and Q700X 


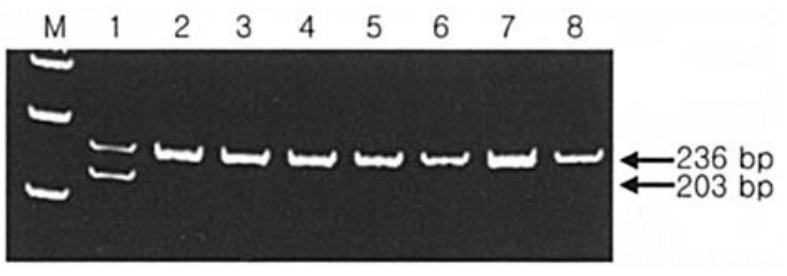

Figure 2. Verification of the 890_922del mutation using PCR-analysis. Lane M shows DNA markers, lane 2 the patient, lane 3 the father, lane 4 the mother, and lanes 4-8 healthy controls. Additional 95 healthy controls showed the same monoallelic pattern (data now shown).

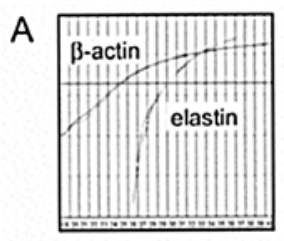

Normal

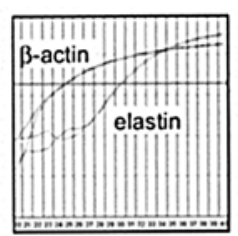

G297_A307del

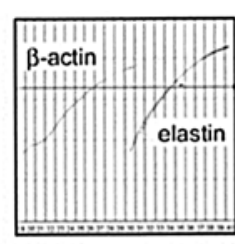

Q700X
B

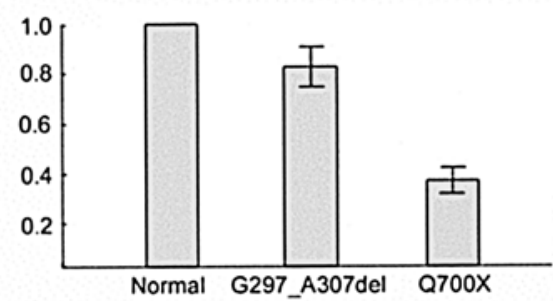

Figure 3. Real-time PCR analysis of SVAS patients. (A) A typical example of real-time PCR analysis using skin fibroblasts from a normal subject and SVAS patients. B-actin was used as an internal control for normalization. (B) In each analysis, the elastin mRNA level was normalized with the B-actin mRNA level and then expressed as a ratio to the normal level. The elastin mRNA level was calculated as the mean \pm standard deviation of assays in quadruplicate.

mutations. The mean mRNA level of elastin, as determined by real-time PCR analysis, was $83 \% \pm 9 \%$ of the normal level in the patient with G297_A308del, whereas in the patient with Q700X the mean mRNA level was only $40 \% \pm 6 \%$ of the normal level (Fig. 3). In contrast, the elastin protein levels, as assessed by Western blot analysis, were significantly reduced in both patients to $42 \% \pm 9 \%$ and $37 \% \pm 10 \%$ of the normal level in patients carrying G297_A308del and Q700X, respectively (Fig. 4).

\section{Discussion}

SVAS shows high penetrance in most kindreds reported so far $(8,12,13-15)$. However, we found a family with an unaffected carrier. The mother of the patient with Q700X showed no signs of SVAS on either the physical or echocardiographic examination. These findings indicate that appropriate cardiac screening and genetic counseling should be available to the unaffected relatives of SVAS patients because unaffected carriers may give birth to severely affected individuals.

In many genes, premature termination codons are known to lead to degradation of mutant mRNAs through a process called nonsense-mediated decay (NMD) $(16,17)$. NMDmediated reduction of expression has been reported in skin
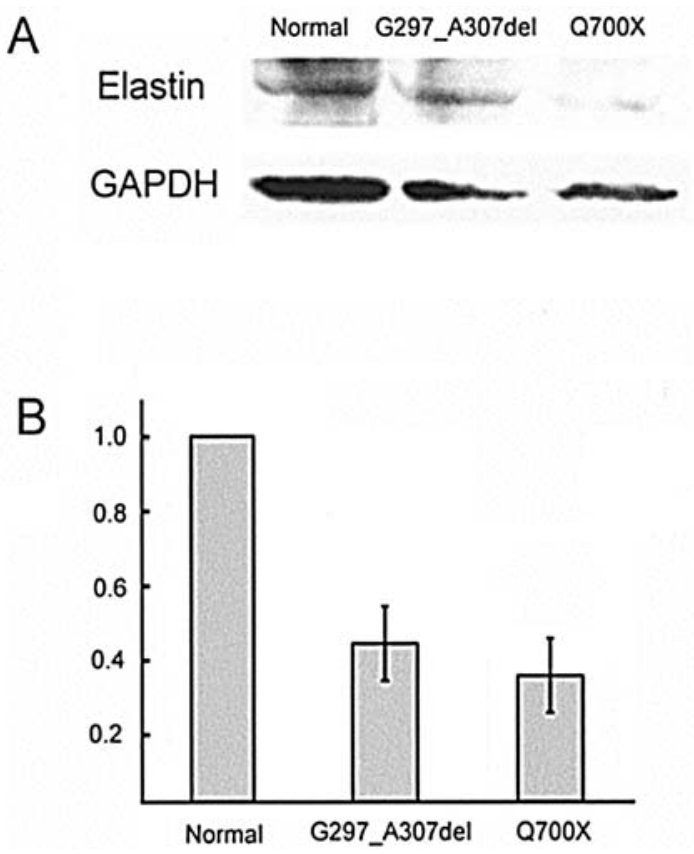

Figure 4. Western blot analysis of SVAS patients. (A) A typical example of Western blot analysis using skin fibroblasts from a normal subject and SVAS patients in quadruplicate assays. GAPDH was used as an internal control for normalization. (B) In each analysis, the elastin protein level was normalized with the GAPDH level and then expressed as a ratio to the normal level. The elastin protein level was calculated as the mean \pm standard deviation of assays in quadruplicate.

fibroblasts and aortic smooth muscle cells of SVAS patients with nonsense mutations in $\operatorname{ELN}(12,13)$. Consistent with these studies $(12,13)$, the newly identified nonsense mutation Q700X also showed significantly reduced expression of elastin at both the mRNA and protein levels in skin fibroblasts from the patient. These results with Q700X further confirm that functional haploinsufficiency of $E L N$, possibly through NMD-mediated degradation of mRNA, is one of the primary pathomechanisms leading to SVAS.

A human tropoelastin protein is composed of 34 domains that are divided into two major types, hydrophobic domains and cross-linking domains. The hydrophobic domains often contain three to six repeats of GVGVP, GGVP or GVGVAP while the cross-linking domains are rich in lysine and alanine (10). The newly identified in-frame deletion mutation G297_A307del results in deletion of GVGTPAAAAAA at codons 297 to 307 in the cross-linking domain encoded by exon 17. In the patient with G297_A307del, the elastin mRNA level was slightly lower than the normal control, but the protein level was reduced to $\sim 50 \%$ of the normal level. These findings possibly suggest that the tropoelastin protein mutated by G297_A307del is subject to proteolytic degradation at a posttranslational level or has an abnormally short half-life, resulting in functional haploinsufficiency of elastin in the patient. Alternatively, the deleted region by G297_A307del may be critical for inter- or intra-molecular cross-linking of tropoelastin monomers, possibly leading to aberrantly polymerized elastin fibers. However, Western blot analysis of skin fibroblasts from the patient with G297_A307del did not detect any aberrant form of tropoelastin corresponding to the 11 amino acid-deleted tropoelastin protein expressed from the 
G297_A307del allele. These results favor the possibility that the tropoelastin mutated by G297_A307del may possess an aberrantly short-half life.

Further detailed functional characterization will be critical for a clearer understanding of how these mutations cause the functional haploinsufficiency of elastin. The functional studies will eventually provide significant insight into the development of therapeutic treatments for the cardiovascular diseases associated with the mutated elastin genes.

\section{Acknowledgements}

We wish to thank all the patients and control members for their participation in this study. This work was supported by Grant 01-PJ10-PG6-01GN15-0001 from the Korean Ministry of Health and Welfare.

\section{References}

1. Wren C, Oslizlok P and Bull C: Natural history of supravalvular aortic stenosis and pulmonary artery stenosis. J Am Coll Cardiol 15: 1625-1630, 1990.

2. Wessel A, Pankau R, Kececioglu D, Ruschewski W and Bursch JH: Three decades of follow-up of aortic and pulmonary vascular lesions in the Williams-Beuren syndrome. Am J Med Genet 52: 297-301, 1994.

3. Ewart AK, Morris CA, Atkinson D, et al: Hemizygosity at the elastin locus in a developmental disorder, Williams syndrome. Nat Genet 5: 11-16, 1993.

4. Francke U: Williams-Beuren syndrome: genes and mechanisms. Hum Mol Genet 8: 1947-1954, 1999.

5. Boeckel T, Dierks A, Vergopoulos A, et al: A new mutation in the elastin gene causing supravalvular aortic stenosis. Am J Cardiol 83: 1141-1143, A9-A10, 1999.
6. Li DY, Toland AE, Boak BB, Atkinson DL, Ensing GJ, Morris CA and Keating MT: Elastin point mutations cause an obstructive vascular disease, supravalvular aortic stenosis. Hum Mol Genet 6: 1021-1028, 1997.

7. Metcalfe K, Rucka AK, Smoot L, et al: Elastin: mutational spectrum in supravalvular aortic stenosis. Eur J Hum Genet 8: 955-963, 2000.

8. Tassabehji M, Metcalfe K, Donnai D, Hurst J, Reardon W, Burch M and Read AP: Elastin: genomic structure and point mutations in patients with supravalvular aortic stenosis. Hum Mol Genet 6: 1029-1036, 1997.

9. Urban Z, Michels VV, Thibodeau SN, Donis-Keller H, Csiszar K and Boyd CD: Supravalvular aortic stenosis: a splice site mutation within the elastin gene results in reduced expression of two aberrantly spliced transcripts. Hum Genet 104: 135-142, 1999.

10. Cleary EG and Gibson MA: Elastic tissue elastin and elastin associated microfibreils. In: Extracellular Matrix. Comper WD (ed). Harwood, Amsterdam, pp95-140, 1991.

11. Rosenbloom J, Abrams WR and Mecham R: Extracellular matrix 4: the elastic fiber. FASEB J 7: 1208-1218, 1993.

12. Urban Z, Michels VV, Thibodeau SN, et al: Isolated supravalvular aortic stenosis: functional haploinsufficiency of the elastin gene as a result of nonsense-mediated decay. Hum Genet 106: $577-588,2000$

13. Urban Z, Riazi S, Seidl TL, et al: Connection between elastin haploinsufficiency and increased cell proliferation in patients with supravalvular aortic stenosis and Williams-Beuren syndrome. Am J Hum Genet 71: 30-44, 2002.

14. Ewart AK, Jin W, Atkinson D, Morris CA and Keating MT: Supravalvular aortic stenosis associated with a deletion disrupting the elastin gene. J Clin Invest 93: 1071-1077, 1994.

15. Curran ME, Atkinson DL, Ewart AK, Morris CA, Leppert MF and Keating MT: The elastin gene is disrupted by a translocation associated with supravalvular aortic stenosis. Cell 73: 159-168, 1993.

16. Frischmeyer PA and Dietz HC: Nonsense-mediated mRNA decay in health and disease. Hum Mol Genet 8: 1893-1900, 1999.

17. Dietz HC: Nonsense mutations and altered splice-site selection. Am J Hum Genet 60: 729-730, 1997. 